\title{
Emotion Regulation Checklist (ERC): Estudos Preliminares da Adaptação e Validação para a Cultura Brasileira
}

\author{
Aline Henriques Reis ${ }^{1}$ \\ Centro de Ciências Humanas e Sociais da Universidade Federal de Mato Grosso do Sul, \\ Campo Grande, MS, Brasil \\ Sérgio Eduardo Silva de Oliveira \\ Denise Ruschel Bandeira \\ Programa de Pós-Graduação em Psicologia da Universidade Federal do Rio Grande do Sul, \\ Porto Alegre, RS, Brasil \\ Nara Côrtes Andrade \\ Programa de Pós-Graduação em Psicologia da Universidade de São Paulo, São Paulo, SP, Brasil \\ Universidade Católica do Salvador, Salvador, BA, Brasil \\ Neander Abreu \\ Instituto de Psicologia da Universidade Federal da Bahia, Salvador, BA, Brasil \\ Tania Mara Sperb \\ Programa de Pós-Graduação em Psicologia da Universidade Federal do Rio Grande do Sul, \\ Porto Alegre, RS, Brasil
}

\begin{abstract}
Resumo
O Emotion Regulation Checklist (ERC) é um instrumento de heterorrelato que se propõe avaliar o nível de regulação emocional de crianças por meio de duas subescalas, a saber, Regulação Emocional (RE) e Labilidade/Negatividade Emocional (L/N). A RE estima a expressão das emoções, empatia e autoconsciência emocional e a $\mathrm{L} / \mathrm{N}$ avalia falta de flexibilidade, desregulação de raiva e labilidade do humor. Objetivou-se traduzir, adaptar e investigar evidências de validade da versão brasileira do ERC. Conduziram-se dois estudos: Estudo I: Tradução e Adaptação do ERC para a cultura brasileira; Estudo II: Investigação de evidências de validade do ERC. Participaram da pesquisa 561 informantes (pais e professoras) de crianças com idades de 3 a 12 anos. Os pressupostos para análise fatorial exploratória foram adequados e a solução bifatorial (RE e $\mathrm{L} / \mathrm{N}$ ) foi a indicada explicando $57 \%$ da variância $(\mathrm{L} / \mathrm{N} \alpha=$ 0,77 e RE $\alpha=0,73$ ). A subescala $\mathrm{L} / \mathrm{N}$ apresentou correlações positivas com medidas de comportamentos problemáticos e a subescala RE apresentou correlações positivas com medidas de habilidades sociais. Os resultados suportaram primeiras evidências de validade do ERC para a cultura brasileira.
\end{abstract}

Palavras-chave: Adaptação, Emotion Regulation Checklist, validação.

\section{Emotion Regulation Checklist (ERC): Preliminary Studies of Cross-Cultural Adaptation and Validation for Use in Brazil}

\begin{abstract}
The Emotion Regulation Checklist (ERC) is an instrument for the hetero-evaluation of the level of emotion regulation of children by means of two scales, Emotion Regulation (ER) and Emotional Lability/
\end{abstract}

Endereço para correspondência: Universidade Federal de Mato Grosso do Sul, Centro de Ciências Humanas e Sociais, Departamento de Ciências Humanas. Cidade Universitária s/n, Campus Universitário, 79070-900, Campo Grande, MS, Brasil, Caixa-postal: 549, Telefone: (67) 3345-7577. E-mail: alinehreis@gmail.com, sergioeduardos.oliveira@gmail.com, deniserbandeira@gmail.com, nara.c.andrade@gmail.com, neandersa@ hotmail.com e sperbt@gmail.com 
Negativity (L/N). ER assesses the expression of emotions, empathy, and emotional self-awareness, while $\mathrm{L} / \mathrm{N}$ assesses the lack of flexibility, anger dysregulation, and mood lability. The aim of this study is to perform the translation and cross-cultural adaptation of the ERC and investigate evidence of the validity of its Brazilian version. Two studies are conducted: Study I - Translation and cross-cultural adaptation of ERC for use in Brazil; and Study II - Investigation of evidence of the validity of the ERC. The sample includes 561 informants (parents and teachers) on children aged 3-12 years old. The exploratory factor analysis (EFA) assumptions are adequate, and the two-factor solution (ER and $\mathrm{L} / \mathrm{N})$ is shown to be the most adequate, explaining $57 \%$ of the variance ( $\mathrm{L} / \mathrm{N} \alpha=.77$ and $\mathrm{ER} \alpha=.73$ ). Subscale $\mathrm{L} / \mathrm{N}$ is positively correlated with measurements of behavioral problems, while subscale ER is positively correlated with measurements of social skills. The present study provides the first evidence of the validity of the ERC for use in the Brazilian context.

Keywords: Adaptation, Emotion Regulation Checklist, validation.

\section{Emotion Regulation Checklist (ERC): Estudios Preliminares de Adaptación y Validación de Cultura de Brasil}

\section{Resumen}

El Emotion Regulation Checklist (ERC) es un instrumento de heterorrelato que propone evaluar el nivel de regulación emocional por medio de dos sub-escalas, Regulación Emocional (RE) y Labilidad/ Negatividad (LN). La RE mide la expresión de las emociones, empatía y autoconsciencia emocional y la $\mathrm{L} / \mathrm{N}$ evalúa la ausencia de flexibilidad, desregulación de rabia y labilidad del humor. Se tuvo como objetivo traducir, adaptar e investigar evidencias de validad de la versión brasileña del ERC. Fueron realizados dos estudios: Estudio I: Traducción y Adaptación del ERC para la cultura brasileña; Estudio II: Investigación de evidencias de validad del ERC. Participaron en esta investigación 561 informantes (padres y profesores) de niños con edad de 3 a 12 años. Los presupuestos para el análisis factorial exploratorio fueron adecuados y la solución bifactorial (RE y L/N) fue la indicada explicando el $57 \%$ de la varianza ( $\mathrm{L} / \mathrm{N} \alpha=.77$ y $\mathrm{RE} \alpha=.73)$. La sub-escala $\mathrm{L} / \mathrm{N}$ presentó correlaciones positivas con medidas de comportamientos problemáticos y la sub-escala RE presentó correlaciones positivas con medidas de habilidades sociales. Los resultados dan soporte a las primeras evidencias de validad del ERC para la cultura brasileña.

Palabras clave: Adaptación, Emotion Regulation Checklist, validación.

A regulação emocional (RE) compreende o manejo adequado da ativação emocional com vistas a um funcionamento social efetivo. Envolve iniciar, manter, modular ou alterar a ocorrência, intensidade ou duração de estados internos e reações fisiológicas relacionadas à emoção. São processos intrínsecos e extrínsecos responsáveis por monitorar, avaliar e modificar reações emocionais, especialmente suas características de intensidade e temporalidade para alcançar metas pessoais (Arango, 2007).

A capacidade de RE é uma habilidade importante para uma vida socioemocional saudável. Saber se autorregular emocionalmente au- menta a probabilidade de aceitação pelos pares e de uma vida social adaptada (Lopes, Salovey, Côté, \& Beers, 2005). A RE possibilita a reflexão do indivíduo sobre situações de conflito e a análise do ponto de vista da outra pessoa com quem se está interagindo. Ela está ligada a dimensões do funcionamento social, tais como empatia e comportamento pró-social (Denham et al., 2012; Eisenberg, 2001). A inabilidade na capacidade de RE é geralmente encontrada em crianças com problemas de comportamento (Andrade, 2013; Izard et al., 2008).

Os estudos sobre RE têm usado diversos instrumentos e metodologias para averigua- 
ção do construto. Existem tarefas para verificar como as crianças identificam e regulam as emoções a partir da apresentação de histórias de conteúdo emocional, e recordação de situações vivenciadas (Davis, Levine, Lench, \& Quas, 2010; Oliveira, Dias, \& Roazzi, 2003). Outro modo de se avaliar a RE é por meio da análise da reação de crianças frente a fotografias, expressões faciais e/ou desenhos (Dias, Vikan, \& Gravas, 2000). Há também a observação da interação mãe-criança (Friedlmeier \& Trommsdorff, 1999). Há ainda formas de investigação da RE que são levadas a efeito por meio de entrevistas (ver os trabalhos de Cruvinel, 2009; Shipman, Edwards, Brown, Swisher, \& Jennings, 2005); tarefas que envolvem atraso de gratificação (Supplee, Skuban, Shaw, \& Prout, 2009; Trentacosta, \& Shaw, 2009); e/ou outros tipos de tarefas (Carthy, Horesh, Apter, Edge, \& Gross, 2010; Kanske, Heissler, Schönfelder, Bongers, \& Wessa, 2011). O monitoramento das emoções eliciadas nas crianças a partir de tarefas pode ser alcançado por medidas de autorrelato, métodos de observação, imageamento cerebral, eletromiografia facial e métodos de detecção de sobressalto (Cacioppo \& Gardner, 1999).

Adrian, Zeman e Veits (2011), em revisão da literatura, analisaram os métodos de avaliação usados por pesquisadores para investigar a RE conforme a idade da criança. Os pesquisadores encontraram que as metodologias de autorrelato foram usadas com frequência significativamente maior com adolescentes e escolares (6-12 anos), quando comparados aos grupos mais jovens (bebês e pré-escolares, cujo método predominante foi o observacional). Outros informantes (pais, professores e pares) foram consultados mais frequentemente no estudo com crianças quando comparados a estudos com adolescentes.

Dentre os instrumentos de heterorrelato para avaliação da RE de crianças, destaca-se o Emotional Regulation Checklist (ERC), um instrumento que contém 24 itens medidos a partir de uma escala tipo Likert de 4 pontos (1. Nunca. 2. Algumas vezes; 3. Frequentemente; 4. Quase Sempre). Pode ser respondido por um adulto familiar à criança, como pai, mãe, cuidador ou professor. Possui duas escalas, sendo que uma delas contém oito itens que avaliam a RE, ou seja, a autoconsciência emocional da criança e a ocorrência de expressividade emocional construtiva. A outra escala com 15 itens mensura a Labilidade/Negatividade Emocional (L/N). Esta escala avalia a falta de flexibilidade, ativação emocional, reatividade, desregulação de raiva e labilidade do humor. A consistência interna das escalas apresentou-se adequada ( $\mathrm{L} / \mathrm{N} \alpha=0,96$; $\operatorname{RE} \alpha=0,83)$ e elas mostraram-se intercorrelacionadas $(r=-0,50, p<0,001)$. A pontuação geral das duas escalas do ERC também foi gerada, como uma única medida de critério para regulação emocional ( $\alpha=0,89$; Shields \& Cicchetti, 1997).

O ERC tem sido amplamente utilizado como uma medida de heterorrelato (preenchida por pais e/ou professores) para averiguar RE e L/N em crianças. Inicialmente designado para crianças de 6 a 12 anos (Shields \& Cicchetti, 1995) tem sido empregado também no estudo das emoções de crianças menores (Morgan, Izard, \& King, 2010; Shields et al., 2001; Shields, Ryan, \& Cichetti, 2001). Outras culturas têm adaptado o ERC, como a Turquia (Batum \& Yagmurlu, 2007) e a China (Chang, Schwartz, Dodge, \& McBride-Chang, 2003).

O ERC tem sido utilizado para verificar a capacidade de RE de crianças com relação à parentalidade (Chang et al., 2003; Ramsden \& Hubbard, 2002) e apego (Borelli et al., 2010), também para verificar a relação entre RE e comportamento agressivo (Chang et al., 2003), sucesso acadêmico (Graziano, Reavis, Keane, \& Calkins, 2007; Leerkes, Paradise, O'Brien, Calkins, \& Lange, 2008) e funcionamento social e comportamental (Ganesalingam, Sanson, Anderson, \& Yeates, 2006; Keane \& Calkins, 2004; Martin, Boekamp, McConville, \& Wheeler, 2010). Estudos utilizaram o ERC para verificar a RE em crianças vítimas e perpetradoras de bullying (Toblin, Schwartz, Hopmeyer Gorman, \& Abou-ezzeddine, 2005), assim como para avaliar o impacto do nascimento prematuro (Clark, Woodward, Horwood, \& Moor, 2008) e da negligência, maus tratos, violência física e/ ou sexual sobre as emoções das crianças (Kim 
\& Cicchetti, 2010; Shields \& Cichetti, 2001; Shipman et al., 2005).

Estudos investigando o efeito do baixo status socioeconômico (Kidwell \& Barnett, 2007; Kliewer, Reid-Quiñones, Shields, \& Foutz, 2009) e de medidas fisiológicas e cerebrais sobre os estados emocionais de crianças também utilizaram o ERC como medida de RE (Borelli et al., 2010; Ganesalingam et al., 2006; Kliewer et al., 2009). Além disso, encontram-se estudos que empregaram o ERC como instrumento padrão-ouro para validação de outros instrumentos (Bulotsky-Shearer \& Fantuzo, 2004; Gouley, Brotman, Huang, \& Shrout, 2008; Zeman, Shipman, \& Penza-Clyve, 2001; Zeman, Cassano, Suveg, \& Shipman, 2010) e como medida pré e pós-teste para programas de intervenção (Izard et al., 2008; Pears, Fischer, \& Bronz, 2007; Suveg, Kendall, Comer, \& Robin, 2006).

Tendo em vista a extensa utilização do ERC, o objetivo da presente pesquisa foi traduzir o Emotion Regulation Checklist (ERC) para o português brasileiro, adaptar os itens para a compreensão de adultos brasileiros com escolaridade mínima de nível fundamental completo e investigar evidências de validade da versão brasileira do ERC. Para tanto, foram conduzidos dois estudos. $\mathrm{O}$ primeiro visou à tradução e adaptação do ERC para a cultura brasileira e o segundo investigou evidências de validade (fatorial e convergente).

\section{Estudo I: Adaptação Linguístico-Cultural do ERC}

O primeiro estudo teve como objetivo traduzir e adaptar os itens do ERC para a cultura brasileira. Para tanto, obteve-se a escala original Emotion Regulation Checklist (Shields \& Cicchetti, 1995), diretamente dos autores, com o devido consentimento para efetivar o processo de tradução, adaptação e validação para o contexto brasileiro. $\mathrm{O}$ protocolo de adaptação baseou-se nas propostas de Beaton, Bombardier, Guillemin, e Ferraz (2000), Cassepp-Borges, Balbinotti, e Teodoro (2010), Geisinger (1994), Oliveira e Bandeira (2011) e Sandoval e Durán (1998).
Primeiramente foram feitas três traduções do inglês para o português. As traduções foram feitas por três juízes independentes, sendo duas professoras da língua inglesa e uma psicóloga bilíngue. As três versões foram analisadas e comparadas pelas autoras Reis e Sperb deste artigo, sendo então formulada uma versão sintetizada com os 24 itens. Essa versão foi apresentada a um comitê de três especialistas selecionados conforme: (a) domínio na língua inglesa; (b) área de atuação (Terapia Cognitivo-Comportamental Infantil); e (c) conhecimento sobre regulação das emoções. Os juízes foram convidados a participar da pesquisa como voluntários e receberam por e-mail um protocolo de tradução contendo informações sobre o ERC, as versões americana e brasileira, e uma tabela para preenchimento contendo cinco informações. Essa tabela continha os 24 itens em português do ERC e nas três primeiras colunas os juízes deveriam julgar em uma escala tipo Likert de cinco pontos $(1=$ "pouquíssima", 2 = "pouca", 3 = "média", 4 = "muita" e 5 = "muitíssima") a clareza dos itens ("Você acredita que a linguagem de cada item é suficientemente clara, compreensível e adequada para professores e pais com ensino fundamental completo? Em que nível?"); a pertinência prática ("Você acredita que os itens propostos são pertinentes para esta população? Em que nível?"); e a relevância teórica ("Você acredita que o conteúdo deste item é representativo do comportamento que se quer medir, ou de uma das dimensões dele, considerando a teoria em questão? Em que nível?"). Na quarta coluna os juízes deveriam indicar a qual dimensão teórica cada item parecia refletir ("Você acredita que este item pertence a que dimensão [fator]? Escreva apenas aquela que melhor representa o item avaliado $[\mathrm{L} / \mathrm{N}$ - labilidade/negatividade; RE - regulação emocional]"). Por fim, a quinta coluna consistia de um espaço para a realização de observações para cada item (Cassepp-Borges et al., 2010; Oliveira \& Bandeira, 2011).

Referente à clareza dos itens, das 24 sentenças do ERC, 20 (83,3\%) foram indicadas como suficientemente claras, com concordância total entre os juízes (os três juízes avaliaram com escores 4 ou 5$)$, e quatro itens $(16,7 \%)$ obtive- 
ram uma concordância parcial (em todos os casos, dois juízes concordaram nos escores e um juiz não). Somente o item 11 obteve uma concordância parcial de que ele se apresentava de forma mediamente clara (dois juízes pontuaram “3” para este item e outro juiz pontuou "4"). Os demais itens receberam concordância parcial indicando a clareza do item. Todas as observações dos juízes foram avaliadas e reformulações foram feitas considerando as sugestões quanto à clareza dos itens.

A avaliação da pertinência prática obteve resultados similares. Dos 24 itens, 83,3\% obtiveram concordância total entre os juízes indicando-os como adequadamente pertinentes. Os demais apresentaram concordância parcial sendo que dois juízes concordaram na adequada pertinência dos itens e um o avaliou como médio ou pouco pertinente. As sugestões dos juízes novamente foram analisadas e reformulações que se mostraram pertinentes foram efetuadas.

Por fim, o julgamento quanto à relevância teórica dos itens para a escala resultou em uma concordância total entre os juízes em 21 (91,3\%) itens. Os três itens restantes tiveram concordância parcial quanto a serem teoricamente relevantes (nos três casos, um dos juízes pontuou " 3 " e os demais "4" ou " 5 "). Novamente foram observadas as sugestões e comentários com a finalidade de melhor representar os itens. A análise da representação do item para seu domínio foi a que apresentou maior divergência.

Após as reformulações efetuadas nos itens a partir do comitê de experts, a versão resultante foi apresentada e discutida em um grupo focal. Os participantes deste grupo foram selecionados por conveniência (Cozby, 2003). O grupo foi formado por quatro mães com idade variando de 28 a 52 anos $(M=35,8 ; D P=11,1)$ e escolaridade de ensino médio e ensino superior. Com este procedimento buscou-se investigar o entendimento dos itens do ERC pelo público alvo. Não houve modificação na escala a partir da aplicação no grupo focal.

A versão resultante do grupo focal foi aplicada em 10 mulheres, com média idade de 34 anos $(D P=6,6)$, e escolaridade de ensino fun- damental completo a superior completo, selecionadas também por conveniência (Cozby, 2003). Este procedimento buscou verificar eventuais problemas de entendimento dos itens ou dificuldades para responder o instrumento. Todos os itens foram claramente compreendidos não ocorrendo novas modificações na escala.

Finalmente, foi feita a retrotradução (backtranslation) para a língua inglesa por um nativo de língua inglesa que reside nos EUA e que domina também a língua portuguesa. A retrotradução foi encaminhada para os autores do instrumento para apreciação. Nenhum item foi modificado pelos autores da escala original e então se obteve a versão experimental da versão brasileira do ERC.

\section{Estudo II: Investigação de Evidências de Validade e Fidedignidade da Versão Experimental do ERC}

O objetivo deste estudo foi investigar a estrutura fatorial da versão brasileira do ERC e verificar suas propriedades psicométricas (consistência interna e evidências de validade convergente).

\section{Método}

\section{Participantes}

A escolha da amostragem foi não probabilística por conveniência (Cozby, 2003). A amostra foi composta por 561 protocolos do ERC respondidos com referência a crianças com idade entre 3 e 12 anos $(M=6,7, D P=2,7) .51,7 \%$ dos protocolos do ERC foram preenchidos pelos pais ( $n$ total de pais $=290)$ e $48,3 \%$ pelos professores $(n$ total de professores $=271)$. Obtiveram-se $o$ ERC de crianças residentes em quatro Estados do Brasil, a saber, Paraná (PR: 51,7\%), Bahia (BA: 26,2\%), Minas Gerais (MG: 14,3\%) e São Paulo (SP: 7,8\%). As crianças do Estado do PR tiveram os protocolos respondidos pelas mães e as crianças dos demais Estados tiveram os protocolos respondidos pelos professores. Do total da amostra, $53,3 \%$ era referente a crianças do sexo 
masculino. Estes protocolos foram utilizados para análise fatorial exploratória. $\mathrm{O}$ tamanho da amostra foi calculado seguindo a razão de vinte casos por item (Hair, Black, Babin, Anderson, \& Tatham, 2006). A Tabela 1 apresenta a caracterização da amostra de forma detalhada.

Tabela 1

Caracterização da Amostra do Estudo II

\begin{tabular}{|c|c|c|c|c|c|}
\hline \multicolumn{6}{|c|}{ Dados das Crianças } \\
\hline & Paraná & Bahia & Minas Gerais & São Paulo & Total \\
\hline \multicolumn{6}{|l|}{ Idade } \\
\hline Mínimo-Máximo & $5-12$ & $3-6$ & $3-6$ & $3-6$ & $3-12$ \\
\hline$M(D P)$ & $8,7(2,1)$ & $4,6(1,1)$ & $4,4(0,9)$ & $4,5(1,1)$ & $6,7(2,7)$ \\
\hline \multicolumn{6}{|l|}{ Sexo } \\
\hline Masculino $f(\%)$ & $166(57,2)$ & $71(48,3)$ & $40(50,0)$ & $22(50,0)$ & $299(53,3)$ \\
\hline Feminino $f(\%)$ & $124(42,8)$ & $76(51,7)$ & $40(50,0)$ & $22(50,0)$ & $262(46,7)$ \\
\hline \multicolumn{6}{|c|}{ Dados dos Respondentes } \\
\hline & Pais & \multicolumn{2}{|c|}{ Professores } & \multicolumn{2}{|c|}{ Total } \\
\hline \multicolumn{6}{|l|}{ Idade } \\
\hline Mínimo-Máximo & $20-59$ & \multicolumn{2}{|c|}{$20-30$} & \multicolumn{2}{|c|}{$20-59$} \\
\hline$M(D P)$ & $36,2(7,1)$ & \multicolumn{2}{|c|}{$26(3,9)$} & \multicolumn{2}{|c|}{$36,1(7,2)$} \\
\hline \multicolumn{6}{|l|}{ Sexo } \\
\hline Masculino $f(\%)$ & $7(2,4)$ & \multicolumn{2}{|c|}{$0(0,0)$} & \multicolumn{2}{|c|}{$7(2,4)$} \\
\hline Feminino $f(\%)$ & $283(97,6)$ & \multicolumn{2}{|c|}{$5(100)$} & \multicolumn{2}{|c|}{$288(97,6)$} \\
\hline
\end{tabular}

Como pode ser observado na Tabela 1, a amostra do PR apresentou uma faixa etária maior, além de uma maior variabilidade de crianças e de respondentes. Além disso, os respondentes do Estado do PR foram todos pais, enquanto que os respondentes dos demais estados foram professores. Os resultados devem ser ponderados de acordo com esses vieses amostrais.

\section{Instrumentos}

Questionário de dados sociodemográficos para identificação de dados de caracterização da amostra para as mães que participaram da pesquisa.

Emotion Regulation Checklist (ERC; Shields \& Cicchetti, 1995): composto por 24 itens heterorrespondidos em escala tipo Likert de quatro pontos segundo a frequência do comportamento $(1=$ "Nunca" a $4=$ "Quase Sempre"). Os itens são distribuídos em duas escalas, a saber, Regulação Emocional (RE) e Labilidade/Negatividade Emocional (L/N). Dados psicométricos e de validade dessa escala podem ser encontrados em Shields e Cicchetti (1995, 1997).

Escala de Habilidades Sociais, Comportamentos Problemáticos e Competência Acadêmica (SSRS-BR; Bandeira, Del Prette, Del Prette, \& Magalhães, 2009): a SSRS-BR inclui medidas de habilidades sociais, de problemas de comportamento e de competência acadêmica de crianças. São três escalas de Habilidades Sociais, uma destinada à criança, outra ao professor e outra aos pais. Dados psicométricos e de validade dessa escala podem ser encontrados em Bandeira et al. (2009).

\section{Procedimentos}

Buscou-se a autorização da direção de escolas públicas e privadas em cidades do Oeste do estado do Paraná e das capitais dos estados da Bahia, Minas Gerais e São Paulo para a condução da pesquisa. A coleta no PR foi realizada de forma coletiva em reuniões de entrega de notas e palestras realizadas no próprio colégio. Os participantes assinaram o Termo de Consenti- 
mento Livre e Esclarecido, receberam um questionário de dados sociodemográficos e o ERC, assim como as instruções para o preenchimento dos instrumentos. A pesquisa foi aprovada no Comitê de Ética em Pesquisa com Humanos do Instituto de Psicologia da Universidade Federal do Rio Grande do Sul (UFRGS; n 21482/2011) e seguiu as medidas éticas pertinentes.

Nos estados da BA, MG e SP, os pais autorizaram a participação através da assinatura do TCLE. Os pesquisadores responsáveis entraram em contato com cada professora, convidando-as a participar da pesquisa e em seguida foi entregue o ERC. As instruções para o seu preenchimento foram explicitadas individualmente e os pesquisadores estiveram disponíveis para o esclarecimento de dúvidas. Esta etapa da pesquisa foi aprovada no Comitê de Ética em Pesquisa da Universidade Federal da Bahia, sob protocolo número 057/2011.

\section{Análise dos Dados}

Considerando o nível de mensuração ordinal das variáveis e a violação do pressuposto de normalidade multivariada (Mardia $=40,449, p<$ 0,001; Mardia, 1970), foi conduzida uma análise fatorial exploratória robusta a partir da matriz de correlações policóricas dos itens (Holgado-Tello, Chacón-Moscoso, Barbero-García, \& Vila-Abad, 2010), com método de extração $M i$ nimum Rank Factor Analysis (MRF; Shapiro \& Berge, 2002) e rotação Promin (Lorenzo-Seva, 1999). O método de extração MRF minimiza a variância comum residual no processo de extração dos fatores, e possibilita a interpretação da proporção da variância comum explicada pelos fatores retidos (Lorenzo-Seva \& Ferrando, 2006). Com a finalidade de evitar a superestimação de fatores comuns, adotou-se como método de retenção e interpretação dos fatores na análise fatorial exploratória o método Hull (Lorenzo-Seva, Timmerman, \& Kiers, 2011). Aplicado às análises fatoriais exploratórias (AFEs), o método Hull tem se apresentado como o melhor método de retenção fatorial já desenvolvido (ver Damásio, 2012; Lorenzo-Seva et al., 2011). O método Hull se aplica da seguinte forma: inicialmente, é determinada a amplitude de fatores a serem avaliados. Para isto, sugere-se utilizar o critério das Análises Paralelas (APs) que indicam a quantidade mínima e máxima de fatores a serem extraídos (Lorenzo-Seva et al., 2011). Posteriormente, se avaliam os índices de adequação de ajuste de todas as soluções fatoriais, bem como os graus de liberdade de cada um dos modelos. A solução fatorial a ser retida refere-se àquela que apresenta o maior valor numérico scree test (st), que se refere a uma fórmula matemática que pondera a relação entre o índice de adequação de ajuste e os graus de liberdade de um modelo, em comparação com um modelo anterior (Damásio, 2012; Lorenzo-Seva et al., 2011). As análises foram conduzidas com o software Factor (v. 9.2; Lorenzo-Seva \& Ferrando, 2006). Para avaliar a validade convergente do ERC, foram realizadas correlações de Pearson entre as suas escalas com as escalas da SSRS-BR (Bandeira et al., 2009).

\section{Resultados}

Para a análise da estrutura fatorial foram conduzidas três análises fatoriais, uma com toda a amostra e as outras duas de acordo com o respondente (pais / professores). Em todos os modelos, a estrutura bifatorial foi confirmada. $\mathrm{O}$ primeiro fator sempre apresentou itens que sinalizavam a dimensão $\mathrm{L} / \mathrm{N}$ e o segundo fator contou com itens indicativos de RE. Observou-se que as EFAs apresentaram resultados similares. Alguns poucos itens mudaram de dimensão sempre alterando o sinal da carga fatorial. Isso deu subsídio para assumir uma AFE para toda a amostra, dada a coerência entre os dados encontrados. Assim, apresentam-se os resultados da AFE para a amostra geral.

Os resultados do Teste de Esfericidade de Bartlett $\left(x^{2}=3889,1 ; g l=276 ; p<0,001\right)$ e do teste Kaiser-Mayer-Olkin para adequação da amostra $(\mathrm{KMO}=0,872)$ indicaram que a matriz de correlações era fatorável. Pelo método de extração MRF foram identificados seis fatores com autovalores $>1$. Entretanto, o método Hull de retenção fatorial demonstrou que uma solução bifatorial seria a mais representativa dos dados (ver Tabela 2). 
Tabela 2

Análise Fatorial Exploratória do ERC $(\mathrm{N}=561)$

\begin{tabular}{|c|c|c|c|c|c|c|}
\hline \multirow[t]{2}{*}{ Itens do ERC } & \multicolumn{2}{|c|}{ AFE 24 itens } & \multicolumn{4}{|c|}{ AFE 23 itens } \\
\hline & $\mathrm{L} / \mathrm{N}$ & $\mathrm{RE}$ & $\mathrm{L} / \mathrm{N}$ & $\mathrm{RE}$ & $\mathrm{r}^{*}$ & $\alpha^{* *}$ \\
\hline 20: Impulsividade & 0,842 & - & 0,833 & - & 0,71 & 0,83 \\
\hline 14: Raiva diante de limites & 0,820 & - & 0,805 & - & 0,73 & 0,83 \\
\hline 08: Explosões de raiva & 0,800 & - & 0,803 & - & 0,74 & 0,83 \\
\hline 13: Explosões de entusiasmo & 0,707 & - & 0,737 & - & 0,65 & 0,83 \\
\hline 22: Entusiasmo intrusivo & 0,698 & - & 0,727 & - & 0,62 & 0,84 \\
\hline 17: Excesso de entusiasmo & 0,695 & - & 0,771 & - & 0,56 & 0,84 \\
\hline $\begin{array}{l}\text { 24: Emoções negativas em convites } \\
\text { para brincar }\end{array}$ & 0,660 & - & 0,609 & - & 0,57 & 0,84 \\
\hline 06: Frustração & 0,642 & - & 0,627 & - & 0,64 & 0,83 \\
\hline 02: Variação de humor & 0,602 & - & 0,588 & - & 0,67 & 0,83 \\
\hline 10: Prazer em ver os outros sofrer & 0,442 & - & 0,449 & - & 0,52 & 0,84 \\
\hline 12: Chorona e agarrada com os adultos & 0,343 & - & 0,327 & - & 0,40 & 0,85 \\
\hline 09: Capaz de adiar gratificação & $-0,307$ & - & $-0,329$ & - & 0,51 & 0,85 \\
\hline 11: Controle de excitação & $-0,306$ & - & $-0,331$ & - & 0,46 & 0,85 \\
\hline 01: Alegria & - & 0,755 & - & 0,782 & 0,58 & 0,72 \\
\hline $\begin{array}{l}\text { 03: Reposta positiva a aproximações } \\
\text { de adultos }\end{array}$ & - & 0,733 & - & 0,763 & 0,65 & 0,71 \\
\hline $\begin{array}{l}\text { 15: Fala sobre estados emocionais } \\
\text { negativos }\end{array}$ & 0,301 & 0,680 & 0,320 & 0,695 & 0,58 & 0,73 \\
\hline 18: Humor apático & - & $-0,656$ & - & $-0,686$ & 0,54 & 0,73 \\
\hline $\begin{array}{l}\text { 07: Resposta positiva a aproximações } \\
\text { de pares }\end{array}$ & - & 0,628 & - & 0,643 & 0,59 & 0,72 \\
\hline 21: Empatia & - & 0,622 & - & 0,558 & 0,50 & 0,74 \\
\hline 05: Recupera-se de emoções negativas & - & 0,512 & - & 0,503 & 0,59 & 0,73 \\
\hline 16: Tristeza e apatia & - & $-0,459$ & - & $-0,489$ & 0,49 & 0,74 \\
\hline $\begin{array}{l}\text { 19: Resposta negativa a aproximações } \\
\text { de pares }\end{array}$ & 0,359 & $-0,410$ & 0,331 & $-0,442$ & 0,51 & 0,73 \\
\hline 04: Troca bem de atividades & - & 0,385 & - & 0,352 & 0,54 & 0,74 \\
\hline $\begin{array}{l}\text { 23: Emoções negativas apropriadas } \\
\text { à demanda }\end{array}$ & 0,330 & 0,368 & & & & \\
\hline Alfa de Cronbach & 0,848 & 0,749 & 0,848 & 0,749 & & \\
\hline Correlação entre fatores & & & & & & \\
\hline Variância comum explicada & & & 56 & & & \\
\hline
\end{tabular}

Nota. ERC = Emotion Regulation Checklist; $\mathrm{AFE}=$ Análise Fatorial Exploratória. $\mathrm{L} / \mathrm{N}=$ Fator 1 "Labilidade/Negatividade"; $\mathrm{RE}=$ Fator 2 "Regulação Emocional”; * correlação Item-Total; ** Alfa de Cronbach se o item for excluído; "-" Carga fatorial menor que 0,300 . 
O primeiro fator contém itens que refletem o construto de $\mathrm{L} / \mathrm{N}$, enquanto que o segundo fator mantém itens relacionados à RE. A estrutura fatorial encontrada sustenta o modelo teórico sugerindo evidências de validade de construto. Todos os itens apresentaram carga fatorial adequada $(\geq 0,30)$. Os itens 15,19 e 23 apresentaram cargas cruzadas. Destacam-se os itens 15 e 23 que apresentaram cargas positivas nos dois fatores. Esse resultado sugere que houve certa confusão na interpretação do item, ora sendo visto como indicador de $\mathrm{L} / \mathrm{N}$ e ora como indicador de RE. Acredita-se que parte da amostra interpretou a palavra "negativo" dos itens como algo ruim, sem contextualizar e/ou compreender o item corretamente. Todavia, dada a carga fatorial do item 15 ter sido limítrofe, decidiu-se por mantê-lo na contagem do fator RE. O item 23 foi excluído na contagem dos fatores. O item 19, por sua vez, foi contado (invertidamente) apenas no fator RE, onde apresentou carga mais saliente.

Uma segunda análise fatorial com os 23 itens (excluindo o item 23) foi feita seguindo os mesmos procedimentos. O objetivo foi verificar se a escala se comportaria da mesma maneira. Os pressupostos para AFE foram adequados (Bartlett: $\chi^{2}=3734,2 ; g l=253 ; p<0,001 ; \mathrm{KMO}$ $=0,880)$ e a solução bifatorial foi a indicada explicando $57 \%$ da variância. As cargas fatoriais foram adequadas (Fator $1-\mathrm{L} / \mathrm{N}$ : variou de 0,33 a 0,83; Fator 2 - ER: variou de 0,35 a 0,78). As correlações entre os itens e o total da escala foram moderadas ( $\mathrm{L} / \mathrm{N}$ : variou de 0,40 a 0,73 ; ER: variou de 0,49 a 0,65$)$. Uma análise de exclusão de itens para melhoramento da consistência interna indicou que nenhuma exclusão favoreceria a consistência das escalas. Desse modo, a escala $\mathrm{L} / \mathrm{N}$ ficou composta por 13 itens, sendo dois itens computados invertidamente. A escala ER por sua vez ficou composta por 10 itens, sendo três contados de forma reversa. A presente estrutura fatorial está de acordo com a postulada teoricamente o que se constitui como uma evidência de validade de construto do ERC. A consistência interna para a escala geral (com 23 itens) também se mostrou adequada $(\alpha=0,86)$. $\mathrm{O}$ exame da exclusão de itens para melhoramento da consistência interna da escala geral também mostrou que a remoção de qualquer item não favoreceria o ERC preenchido pelos pais e professores.

Para analisar a fidedignidade entre avaliadores, verificou-se a correlação entre os escores das escalas do ERC pontuadas pelos pais e professores em uma subamostra de 38 crianças. Os resultados não apoiaram evidências de precisão entre avaliadores (L/N: $r=0,18$; ER: $r=0,24$; ERC Total: $r=0,22$ : níveis de significância estatística maiores que 0,05$)$. O teste $t$ para amostras pareadas indicou não haver diferença entre as médias das crianças na escala RE pontuada pelos pais e professores (Professores: $M=31,5, D P$ $=4,0$; Pais: $M=30,7, D P=5,0 ; t[37]=-0,871$, $p=0,390)$. Para $\mathrm{L} / \mathrm{N}$ as médias foram diferentes (Professores: $M=22,1, D P=5,7$; Pais: $M=$ $28,2, D P=6,3 ; t[37]=4,883, p<0,001)$. Para o escore geral também se encontrou diferença estatisticamente significativa (Professores: $M=$ $74,4, D P=8,7$; Pais: $M=67,5, D P=9,5 ; t[37]$ $=3,741, p=0,001)$. Esses resultados sugerem a elaboração de normas de interpretação de acordo com o respondente (pais ou professores).

Para essa subamostra de crianças também os pais, professores e as próprias crianças preencheram a SSRS-BR. A Tabela 3 apresenta os resultados das correlações entre os escores da versão brasileira do ERC e a SSRS-BR segundo o tipo de respondente.

Os resultados mostraram que o ERC e a SSRS-BR preenchidas pelos pais indicaram evidências de validade. A escala $\mathrm{L} / \mathrm{N}$ apresentou relação positiva com problemas de comportamento hiperativos, externalizantes e internalizantes. Crianças avaliadas pelos pais com altos escores em $\mathrm{L} / \mathrm{N}$ foram avaliadas como tendo menores habilidades de autocontrole e civilidade e de autocontrole passivo. As crianças avaliadas pelos pais com habilidades em RE foram observadas com maiores habilidades sociais, exceto em cooperação e autocontrole passivo. Entre as percepções dos professores, a L/N e RE relacionaram-se com responsabilidade, em direção negativa e positiva respectivamente. Observou-se apenas uma relação significativa entre a autoavaliação das crianças e as medidas do ERC respondidas pelos pais. Observou-se que as crianças que se percebiam menos assertivas foram aquelas ava- 
Tabela 3

Correlações entre o ERC e a SSRS-BR $(\mathbf{N}=38)$

\begin{tabular}{|c|c|c|c|c|c|c|c|}
\hline \multirow{3}{*}{ Respondente } & \multirow{3}{*}{ Variáveis } & \multicolumn{6}{|c|}{ Respondentes } \\
\hline & & \multicolumn{3}{|c|}{ Professores } & \multicolumn{3}{|c|}{ Pais } \\
\hline & & $\mathbf{L} / \mathbf{N}$ & $\mathbf{R E}$ & Total & $\mathbf{L} / \mathbf{N}$ & RE & Total \\
\hline \multicolumn{8}{|c|}{ Habilidades Sociais } \\
\hline & Cooperação & 0,05 & 0,24 & 0,08 & $-0,16$ & 0,29 & 0,28 \\
\hline & Amabilidade & $-0,16$ & 0,30 & 0,25 & $-0,20$ & $0,46 * *$ & $0,39 *$ \\
\hline & Asserção & $-0,26$ & 0,28 & 0,27 & $-0,24$ & $0,56 * * *$ & $0,46 * *$ \\
\hline & Iniciativa/Desenvoltura Social & $-0,11$ & 0,16 & 0,13 & $-0,20$ & $0,50 * * *$ & $0,39 *$ \\
\hline \multirow{2}{*}{ Pais } & Autocontrole e Civilidade & $-0,05$ & 0,01 & 0,04 & $-0,55 * * *$ & $0,46^{* *}$ & $0,59 * * *$ \\
\hline & Autocontrole Passivo & 0,12 & $-0,11$ & $-0,11$ & $-0,62 * * *$ & 0,29 & $0,55 * * *$ \\
\hline
\end{tabular}

Comportamentos Problemáticos

$\begin{array}{lcccccc}\text { Hiperatividade } & 0,41 * & -0,31 & -0,47 * * & 0,64 * * * & -0,43 * * & -0,60 * * * \\ \text { Problemas Externalizantes } & 0,40 * & -0,18 & -0,40 * & 0,70 * * * & -0,38^{*} & -0,65 * * * \\ \text { Problemas Internalizantes } & 0,42 * * & -0,16 & -0,32 & 0,35 * & -0,56 * * * & -0,53 * * *\end{array}$

Habilidades Sociais

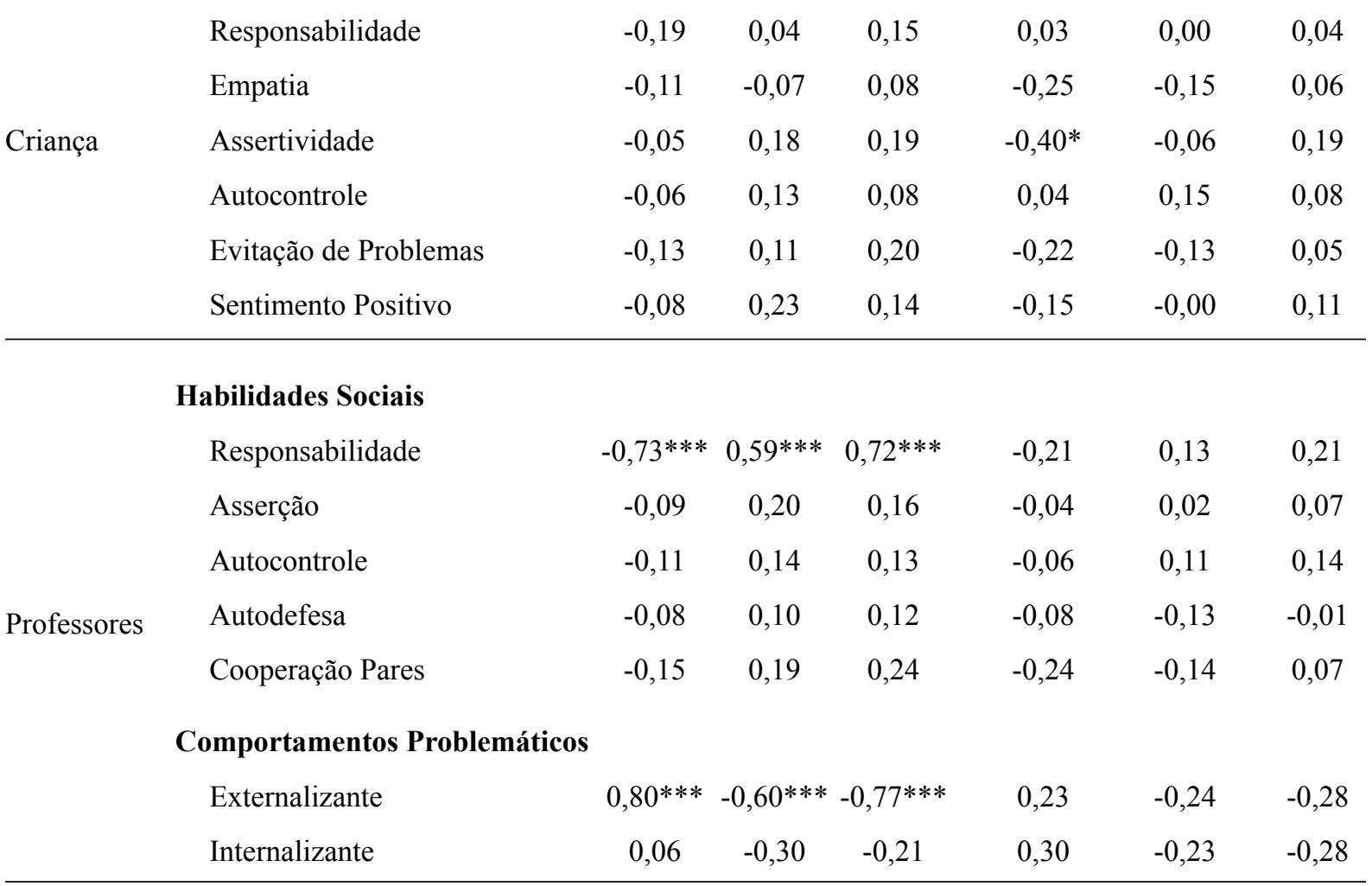

Nota. ERC = Emotion Regulation Checklist; SSRS-BR = Escala de Habilidades Sociais, Comportamentos Problemáticos e Competência Acadêmica. L/N = Labilidade/Negatividade Emocional; RE = Regulação Emocional.

$* \mathrm{p} \leq 0,05 ; * * \mathrm{p} \leq 0,01 ; * * * \mathrm{p} \leq 0,001$. 
liadas pelos pais com maior grau de L/N. Esses resultados indicam que a validade convergente do ERC parece depender do tipo de respondente. Observa-se uma convergência entre a percepção dos pais nos diferentes instrumentos. Uma convergência parcial foi encontrada entre a percepção dos professores nos dois instrumentos acerca da capacidade de regulação emocional das crianças e suas competências sociais.

Verificaram-se as diferenças das médias das escalas pelos Estados de residência das crianças (BA, MG e SP) cujas ERC foram preenchidos pelas professoras, ao se considerar a diferença já encontrada entre os tipos de respondente (pais ou professores). Os resultados mostraram que as escalas L/N, RE e ERC Total diferenciaram significativamente apenas para o Estado de SP. Em $\mathrm{SP}$ as crianças foram avaliadas com maiores ha- bilidades de RE e com menor $\mathrm{L} / \mathrm{N}$ que as crianças da BA e MG. O reduzido tamanho amostral pode ter tido efeito sobre esse resultado. Assim, sugerem-se investigações normativas mais extensas por regiões.

Para verificar a diferença por sexo foram considerados os efeitos do Estado de residência e do tipo de respondente sobre o escores das crianças, conforme já encontrados neste estudo. Não foram encontradas diferenças estatisticamente significativas entre as crianças dos Estados do PR e SP. Entre as crianças da BA, observou-se que os meninos apresentaram menor habilidade de RE. Entre as crianças de MG as diferenças foram para a escala $\mathrm{L} / \mathrm{N}$, sendo que os meninos apresentaram médias mais altas, e para o escore global do ERC, tendo os meninos apresentando escores mais baixos. A Tabela 4 sumariza os resultados obtidos.

Tabela 4

Diferenças por Sexo

\begin{tabular}{|c|c|c|c|c|}
\hline Estado & Escala & $\begin{array}{c}\text { Meninos } \\
M(D P)\end{array}$ & $\begin{array}{c}\text { Meninas } \\
M(D P)\end{array}$ & Diferença \\
\hline \multirow{5}{*}{ Paraná } & $\mathrm{L} / \mathrm{N}$ & $27,0(5,7)$ & $26,8(5,7)$ & $t(288)=0,388, p=0,698$ \\
\hline & $\mathrm{RE}$ & $31,3(4,9)$ & $32,0(4,1)$ & $t(288)=-1,276, p=0,203$ \\
\hline & Total & $69,3(8,5)$ & $70,3(8,3)$ & $t(288)=-0,955, p=0,341$ \\
\hline & $\mathrm{L} / \mathrm{N}$ & $23,9(7,2)$ & $21,6(6,4)$ & $t(145)=2,089, p=0,038$ \\
\hline & $\mathrm{RE}$ & $31,4(4,1)$ & $32,9(3,7)$ & $t(145)=-2,344, p=0,020$ \\
\hline \multirow[t]{3}{*}{ Bahia } & Total & $72,5(9,8)$ & $76,3(8,5)$ & $t(145)=-2,541, p=0,012$ \\
\hline & $\mathrm{L} / \mathrm{N}$ & $24,0(7,0)$ & $19,9(5,1)$ & $t(78)=3,054, p=0,003$ \\
\hline & RE & $32,1(4,5)$ & $33,5(4,1)$ & $t(78)=-1,429, p=0,157$ \\
\hline \multirow[t]{3}{*}{ Minas Gerais } & Total & $73,1(9,5)$ & $78,6(7,5)$ & $t(78)=-2,874, p=0,005$ \\
\hline & $\mathrm{L} / \mathrm{N}$ & $17,4(5,2)$ & $17,5(5,7)$ & $t(42)=-0,055, p=0,956$ \\
\hline & $\mathrm{RE}$ & $36,8(4,6)$ & $36,0(4,5)$ & $t(42)=0,565, p=0,575$ \\
\hline São Paulo & Total & $84,4(9,3)$ & $83,5(9,6)$ & $t(42)=0,303, p=0,764$ \\
\hline
\end{tabular}

Nota. $\mathrm{L} / \mathrm{N}=$ Labilidade/Negatividade; RE = Regulação Emocional; $\mathrm{M}=$ Média; DP = Desvio-padrão.

Por fim, foi verificada a relação entre os escores das escalas do ERC com a idade das crianças. Para as correlações foram controlados os efeitos do sexo, da região geográfica de moradia e o tipo de respondente. A única correlação es- tatisticamente significativa encontrada foi entre as crianças do PR na escala RE $(r=-0,12, p=$ $0,037)$. Contudo, a magnitude da relação é muito baixa. As crianças foram agrupadas em cinco faixas etárias (3-4 anos, 5-6 anos, 7-8 anos, 9-10 
anos, 11-12 anos) e investigaram-se as diferenças de médias entre as faixas, controlando os mesmo efeitos. Os resultados indicaram que as diferenças não foram estatisticamente significativas.

\section{Discussão}

\section{Estrutura Fatorial e Consistência Interna}

A investigação da estrutura fatorial da versão brasileira do ERC resultou em um modelo bifatorial, conforme o modelo teórico e empírico apresentado na literatura (Batum \& Yagmurlu, 2007; Melo, 2005; Shields \& Cicchetti, 1998). O primeiro fator, com 13 itens, é constituído por indicadores de $\mathrm{L} / \mathrm{N}$. Esse construto refere-se a demonstrações afetivas culturalmente inapropriadas, forte reatividade e desregulação da raiva, intensidade emocional e emoções positivas e negativas desreguladas. Indivíduos com labilidade emocional são propensos a explosões de raiva, baixa tolerância à frustração, impulsividade e variabilidade emocional com rápida transição de emoções positivas para negativas (Shields \& Cicchetti, 1997, 1998).

O segundo fator, com 10 itens, é formado por sentenças que sinalizam a vivência de RE. Esse construto é entendido como a autoconsciência emocional da criança, a expressão emocional socialmente apropriada e empatia. Indivíduos que são bem regulados emocionalmente identificam e comunicam corretamente as próprias emoções, e expressam e manejam emoções negativas considerando os objetivos pessoais e as pistas do contexto (Gross \& Thompson, 2009; Shields \& Cicchetti, 1997, 1998).

Foram encontrados poucos estudos psicométricos do ERC, apesar de ser um instrumento amplamente utilizado (ver, por exemplo, Ramsden \& Hubbard, 2002; Toblin et al., 2005). Dentre os estudos psicométricos encontrados, observou-se que a estrutura fatorial da presente pesquisa mostrou-se semelhante às encontradas na literatura (Melo, 2005; Shields \& Cichetti, 1995). A consistência interna das escalas mostrou-se adequada. Avalia-se, com base em Anastasi e Urbina (2000), que a ERC apresenta, portanto, além de indicadores de precisão, evi- dências de validade de construto dada a correlação dos indicadores internos das escalas.

\section{Evidências de Validade de Construto}

Os resultados referentes à validade de construto sugeriram evidências parciais de validade. Observou-se convergência entre a percepção dos pais acerca das habilidades sociais dos filhos e suas habilidades de RE. Entre os professores o resultado foi menos expressivo e menor ainda quando comparados os resultados das percepções dos pais e professores com as autoavaliações das crianças sobre suas habilidades sociais.

A capacidade de RE tende a se refletir nas interações sociais. Habilidades de RE relacionaram-se a diversos indicadores da qualidade da interação social com os pares. Crianças com altos escores em RE foram vistas mais favoravelmente pelos pares e perceberam a si mesmas como mais sensíveis e pró-sociais nas relações interpessoais (Lopes et al., 2005). A desregulação da emoção tem se mostrado associada à má adaptação psicológica, geralmente moderada pelo grau no qual a criança se engaja em interações sociais (Rubin, Coplan, Fox, \& Calkins, 1995).

Ao tomar como base os resultados da presente pesquisa, e considerar que o ERC é preenchido por um adulto responsável (pais, familiares, professores, etc.) referente ao comportamento de uma criança, entende-se que a escala está sujeita à experiência e ao nível de conhecimento do respondente com relação à criança. Assim, o ERC reflete a percepção do respondente, e não somente o comportamento da criança. Esse fenômeno é observado em outros instrumentos heterorrespondidos, como o caso do Child Behavior Checklist (CBCL; ver, por exemplo, Rocha, Ferrari, \& Silvares, 2011).

Considerando essas diferenças perceptivas entre observadores, observou-se maior correlação entre os escores dados pelos pais nas escalas ERC e SSRS-BR. Esse achado sugere validade de conteúdo da escala de acordo com a percepção parental. Os resultados da presente pesquisa mostraram que as crianças, com adequada capacidade de RE, eram mais amáveis, assertivas e desenvoltas nas interações sociais. Elas também apresentaram maior capacidade de autocontrole. 
Crianças com maior $\mathrm{L} / \mathrm{N}$ apresentaram menor propensão a apresentar comportamentos hiperativos, problemas externalizantes e internalizantes. A literatura revela, de forma congruente com esse achado, que crianças com comportamentos externalizantes são propensas a apresentar altos níveis de raiva, tristeza e dificuldades em autocontrole. Ainda, mostram dificuldade em controlar a demonstração de emoções negativas. Por sua vez, crianças com problemas internalizantes tendem a apresentar tristeza e baixa impulsividade e dificuldade em regular as emoções de tristeza e ansiedade (Eisenberg et al., 2001). A RE pode auxiliar crianças de alto risco a diminuir problemas de comportamentos na primeira infância (Hill, Degnan, Calkins, \& Keane, 2006).

Considerando a percepção dos professores nessas duas medidas, observou-se que a habilidade de RE está positivamente relacionada à responsabilidade. Esta é entendida como ações que indicam comprometimento com as atividades e com as pessoas do ambiente escolar, por exemplo, seguir as instruções do professor, engajar-se nas tarefas (Bandeira et al., 2009). Dessa forma, crianças capazes de regular suas emoções tendem a obter maior sucesso acadêmico mesmo após controlar a influência de outras variáveis cognitivas, incluindo o desempenho escolar e autoeficácia acadêmica (Gumora \& Arsenio, 2002).

Os achados entre os professores ainda indicaram uma relação inversa entre habilidade de RE e comportamentos externalizantes. Os problemas de comportamentos externalizantes são caracterizados por agressão física ou verbal, violação de regras e baixo autocontrole da raiva manifestados diretamente no ambiente (Bandeira et al., 2009). Rocha et al. (2011) observaram maior concordância entre informações referentes a comportamentos externalizantes, devido à característica fenomenológica desse tipo de problema. Os problemas externalizantes são manifestados numa forma facilmente observável, dada sua característica direcionada ao ambiente. $\mathrm{O}$ fato de não se ter encontrado relação significativa entre os indicadores de RE e comportamentos internalizantes pelos professores, pode ser, portanto, devido à manifestação fenomenológica desse último tipo.
Por fim, foi observada somente uma relação inversa entre a autopercepção das crianças e a percepção dos pais no que se refere à assertividade e à $\mathrm{L} / \mathrm{N}$. As crianças que se viam mais assertivas eram vistas pelos pais com menor problema de L/N. De uma maneira geral, habilidades de $\mathrm{RE}$ tendem a se correlacionar positivamente à competência social (Lopes et al., 2004). Correlações negativas foram encontradas entre simpatia e emocionalidade negativa (estresse, raiva e frustração; Eisenberg et al., 1996).

\section{Limitações do Estudo}

O presente manuscrito se propôs a reportar resultados preliminares da adaptação e validação do ERC para a cultura brasileira. Dessa forma, as limitações aqui encontradas, se modificadas, poderiam contribuir para uma estimação mais acurada das propriedades psicométricas da versão brasileira do ERC. A desproporção das médias etárias das crianças avaliadas, segundo o tipo de avaliador e a região geográfica, impossibilita maior precisão na interpretação dos resultados encontrados. Dessa forma, controlando essas variáveis, poderão ser verificados com maior precisão os efeitos da faixa etária das crianças e do tipo de observador (pais versus professores) sobre os escores nas escalas do ERC. As diferenças regionais também não foram passíveis de verificação conclusiva devido à falta de controle da faixa etária, do tipo de respondente e do tamanho amostral em todas as regiões estudadas. A falta de critérios externos para seleção da amostra e a não aleatorização da mesma também impossibilitaram a elaboração de interpretações mais precisas para as escalas. Além disso, a ausência de grupos-critério impossibilitou verificar a capacidade discriminativa das escalas do ERC. O emprego de um instrumento de triagem e classificação de grupos contrastantes, como o CBCL, por exemplo, poderia auxiliar na investigação do poder discriminativo do ERC.

\section{Considerações Finais}

A análise das características psicométricas do ERC quanto à sua estrutura fatorial corroboraram a bidimensionalidade da mesma, conver- 
gindo para os fatores teóricos de Regulação de Emoção (RE) e Labilidade/Negatividade (L/N). Como previsto, as subescalas do ERC relacionaram-se com diversas dimensões do comportamento social das crianças, tais como assertividade, habilidades de autocontrole e civilidade, e de autocontrole passivo. Observaram-se relações também entre o ERC e comportamentos hiperativos, externalizantes e internalizantes. Essas relações indicaram evidências de validade de tipo convergente para o ERC.

As diferenças encontradas entre os escores do ERC no que diz respeito ao tipo de respondente, a região geográfica de residência da criança e ao sexo da criança sugerem que a normatização dos escores considere estes fatores. A análise de consistência interna do ERC e de suas subescalas também se mostrou adequada. Estes dados indicam que esta escala pode ser um importante método de avaliação da capacidade de regulação emocional de crianças, aumentando o escopo de instrumentos disponíveis no Brasil.

\section{Referências}

Adrian, M., Zeman, J., \& Veits, G. (2011). Methodological implications of the affect revolution: A 35-year review of emotion regulation assessment in children. Journal of Experimental Child Psychology, 110(2), 171-197. doi:10.1016/j. jecp.2011.03.009

Anastasi, A., \& Urbina, S. (2000). Testagem psicológica. Porto Alegre, RS: Artes Médicas.

Andrade, N. (2013). Adaptação transcultural e validação do Teste de Conhecimento Emocional: Avaliação neuropsicológica das emoções (Dissertação de mestrado, Instituto de Psicologia, Universidade Federal da Bahia, Salvador, BA, Brasil).

Arango, M. I. R. (2007). Regulación emocional y competência social em la infância. Diversitas: Perspectivas em Psicologia, 3(2), 349-363. Recuperado em http://pepsic.bvsalud.org/pdf/diver/v3n2/v3n2a14.pdf

Bandeira, M., Del Prette, Z. A. P., Del Prette, A., \& Magalhães, T. (2009). Validação das escalas de habilidades sociais, comportamentos problemáticos e competência acadêmica (SSRS-BR) para o ensino fundamental. Psicologia: Teoria e Pesquisa, 25(2), 271-282. doi:10.1590/S010237722009000200016.

Batum, P., \& Yagmurlu, B. (2007). What counts in externalizing behaviors? The contributions of emotion and behavior regulation. Current Psychology: Developmental Learning Personality Social, 25(4), 272-294. doi:10.1007/bf02915236

Beaton, D. E., Bombardier, C., Guillemin, F., \& Ferraz, M. B. (2000). Guidelines for the process of cross-cultural adaptation of Self-Report Measures. Spine, 25(24), 3186-3191. doi:10.1097/00007632-200012150-00014

Borelli, J. L., Crowley, M. J., David, D. H., Sbarra, D. A., Anderson, G. M., \& Mayes, L. C. (2010). Attachment and emotion in school-aged children. Emotion, 10(4), 475-485. doi:10.1037/ a0018490

Bulotsky-Shearer, R., \& Fantuzzo, J. W. (2004). Adjustment scales for preschool intervention: Extending validity and relevance across multiple perspectives. Psychology in the Schools, 41(7), 725-736. doi:10.1002/pits.20018

Cacioppo, J. T., \& Gardner, W. L. (1999). Emotion. Annual Review of Psychology, 50(1), 191-214. doi:10.1146/annurev.psych.50.1.191

Carthy, T., Horesh, N., Apter, A., Edge, M. D., \& Gross, J. J. (2010). Emotional reactivity and cognitive regulation in anxious children. Behavior Research Therapy, 48(5), 384-93. doi:10.1016/j. brat.2009.12.013

Cassepp-Borges, V., Balbinotti, M. A. A., \& Teodoro, M. L. M. (2010). Tradução e validação de conteúdo: Uma proposta para a adaptação de instrumentos. In L. Pasquali (Ed.), Instrumentação psicológica: Fundamentos e práticas (pp. 506-520). Porto Alegre, RS: Artmed.

Chang, L., Schwartz, D., Dodge, K. A., \& McBrideChang, C. (2003). Harsh parenting in relation to child emotion regulation and aggression. Journal of Family Psychology, 17(4), 598-606. doi:10.1037/0893-3200.17.4.598

Clark, C. A. C., Woodward, L. J., Horwood, L. J., \& Moor, S. (2008). Development of emotional and behavioral regulation in children born extremely preterm and very preterm: Biological and social influences. Child Development, 79(5), 14441462. doi:10.1111/j.1467-8624.2008.01198.x

Cozby, P. C. (2003). Métodos de pesquisa em ciências do comportamento. São Paulo, SP: Atlas. 
Cruvinel, M. (2009). Correlatos cognitivos e psicossociais de crianças com e sem sintomas depressivos (Tese de doutorado, Universidade Estadual de Campinas, SP, Brasil).

Damásio, B. F. (2012). O uso da análise fatorial exploratória em Psicologia. Avaliação Psicológica, 11(2), 213-228. Recuperado em http:// pepsic.bvsalud.org/scielo.php? script $=$ sci arttext\&pid=S1677-04712012000200007\&lng= $\mathrm{pt \& t} \operatorname{lng}=\mathrm{pt}$

Davis, E. L., Levine, L. J., Lench, H. C., \& Quas, J. A. (2010). Metacognitive emotion regulation: Children's awareness that changing thoughts and goals can alleviate negative emotions. Emotion, 10(4), 498-510. doi:10.1037/a0018428

Denham, S. A., Bassett, H. H., Mincic, M., Kalb, S., Way, E., Wyatt, T., \& Segal, Y. (2012). Socialemotional learning profiles of preschoolers' early school success: A person-centered approach. Learning and Individual Differences, 22(2), 178-189. doi:10.1016/j.lindif.2011.05.001

Dias, M. G. B. B., Vikan, A., \& Gravas, S. (2000). Tentativa de crianças em lidar com as emoções de raiva e tristeza. Estudos de Psicologia (Natal), 5(1), 49-70. doi:10.1590/S1413$-294 X 2000000100004$

Eisenberg, N. (2001). The core and correlates of affective social competence. Social Development, 10, 120-124. doi:10.1111/1467-9507.00151

Eisenberg, N., Cumberland, A., Spinrad, T. L., Fabes, R. A., Shepard, S. A., Reiser, M., ...Guthrie, I. K. (2001). The relations of regulation and emotionality to children's externalizing and internalizing problem behavior. Child Development, 72(4), 1112-1134. doi:10.1111/1467-8624.00337

Eisenberg, N., Fabes, R. A., Murphy, B., Karbon, M., Smith, M., \& Maszk, P. (1996). The relations of children's dispositional empathy-related responding to their emotionality, regulation, and social functioning. Developmental Psychology, 32(2), 195-209. doi:10.1037/00121649.32.2.195

Friedlmeier, W., \& Trommsdorff, G. (1999). Emotion regulation in early childhood: A cross-cultural comparison between German and Japanese toddlers. Journal of CrossCultural Psychology, 30(6), 684-711. doi:10.1177/0022022199030006002

Ganesalingam, K., Sanson, A., Anderson, V., \& Yeates, K. O. (2006). Self-regulation and social and behavioral functioning following childhood traumatic brain injury. Journal International Neuropsychological Society, 12(5), 609-621. doi:10.1017/S1355617706060796

Geisinger, K. F. (1994). Cross-cultural normative assessment: Translation and adaptation issues influencing the normative interpretation of assessments instruments. Psychological Assessment, 6, 304-312. doi:10.1037/1040-3590.6.4.304

Gouley, K. K., Brotman, L. M., Huang, K.-Y., \& Shrout, P. E. (2008). Construct validation of the social competence scale in preschool-age children. Social Development, 17(2), 380-398. doi:10.1111/j.1467-9507.2007.00430.x

Graziano, P. A., Reavis, R. D., Keane, S. P., \& Calkins, S. D. (2007). The role of emotion regulation in children's early academic success. Journal of School Psychology, 45(1), 3-19. doi:10.1016/j.jsp.2006.09.002

Gross, J. J., \& Thompson, R. A. (2009). Emotion regulation: Conceptual foundations. In J. J. Gross (Ed.), Handbook of emotion regulation (pp. 3-24). New York: The Guilford Press.

Gumora, G., \& Arsenio, W. F. (2002). Emotionality, emotion regulation, and school performance in middle school children. Journal of School Psychology, 40(5), 395-413. doi:10.1016/S00224405(02)00108-5

Hair, J. F., Jr., Black, W. C., Babin, B. J., Anderson, R. E., \& Tatham, R. L. (2006). Multivariate data analysis ( $6^{\text {th }}$ ed.). Upper Saddle River, NJ: Pearson Prentice Hall.

Hill, A. L., Degnan, K. A., Calkins, S. D., \& Keane, S. P. (2006). Profiles of externalizing behavior problems for boys and girls across preschool: The roles of emotion regulation and inattention. Developmental Psychology, 42(5), 913-928. doi:10.1037/0012-1649.42.5.913

Holgado-Tello, F., Chacón-Moscoso, S., BarberoGarcía, I., \& Vila-Abad, E. (2010). Polychoric versus Pearson correlations in exploratory and confirmatory factor analysis of ordinal variables. Quality and Quantity, 44(1), 153-166. doi:10.1007/s11135-008-9190-y

Izard, C. E., King, K. A., Trentacosta, C. J., Morgan, J. K., Laurenceau, J.-P., Krauthamer-Ewing, E. S., \& Finlon, K. J. (2008). Accelerating the development of emotion competence in Head Start children: Effects on adaptive and maladaptive behavior. Development and Psy- 
chopathology, 20(1), 369-397. doi:10.1017/ S0954579408000175

Kanske, P., Heissler, J., Schönfelder, S., Bongers, A., \& Wessa, M. (2011). How to regulate emotion? Neural networks for reappraisal and distraction. Cerebral Cortex, 21(6), 1379-1388. doi:10.1093/cercor/bhq216

Keane, S., \& Calkins, S. (2004). Predicting kindergarten peer social status from toddler and preschool problem behavior. Journal of Abnormal Child Psychology, 32(4), 409-423. doi:10.1023/ b:jacp.0000030294.11443.41

Kidwell, S. L., \& Barnett, D. (2007). Adaptive emotion regulation among low-income African American children. Merrill-Palmer Quarterly, 53(2), 155-183. doi:10.1353/mpq.2007.0011

Kim, J., \& Cicchetti, D. (2010). Longitudinal pathways linking child maltreatment, emotion regulation, peer relations, and psychopathology. Journal of Child Psychology and Psychiatry, 51(6), 706-716. doi:10.1111/j.1469-7610.2009.02202

Kliewer, W., Reid-Quiñones, K., Shields, B. J., \& Foutz, L. (2009). Multiple risks, emotion regulation skill, and cortisol in low-income African American youth: A prospective study. Journal of Black Psychology, 35(1), 24-43. doi:10.1177/0095798408323355

Leerkes, E. M., Paradise, M., O’Brien, M., Calkins, S. D., \& Lange, G. (2008). Emotion and cognition processes in preschool children. MerrillPalmer Quarterly, 54(1), 102-124. doi:10.1353/ mpq.2008.0009

Lopes, P. N., Brackett, M. A., Nezlek, J. B., Schütz, A., Sellin, I., \& Salovey, P. (2004). Emotional intelligence and social interaction. Personality and Social Psychology Bulletin, 30(8), 10181034. doi:10.1177/0146167204264762

Lopes, P. N., Salovey, P., Côté, S., \& Beers, M. (2005). Emotion regulation abilities and the quality of social interaction. Emotion, 5(1), 113 118. doi:10.1037/1528-3542.5.1.113

Lorenzo-Seva, U. (1999). Promin: A method for oblique factor rotation. Multivariate Behavioral Research, 34(3), 347-356. doi:10. 1207?S14327906MBR3403_3

Lorenzo-Seva, U., \& Ferrando, P. J. (2006). Factor: A computer program to fit the exploratory factor analysis model. Behavior Research Methods, 38(1), 88-91. doi:10.3758/BF03192753
Lorenzo-Seva, U., Timmerman, M. E., \& Kiers, H. A. L. (2011). The Hull method for selecting the number of common factors. Multivariate Behavioral Research, 46(2), 340-364. doi:10.1080/00 273171.2011 .564527

Mardia, K. V. (1970). Measures of multivariate skewness and kurtosis with applications. Biometrika, 57(1), 519-530. doi:10.1093/biomet/57.3.519

Martin, S., Boekamp, J., McConville, D., \& Wheeler, E. (2010). Anger and sadness perception in clinically referred preschoolers: Emotion processes and externalizing behavior symptoms. Child Psychiatry \& Human Development, 41(1), 3046. doi:10.1007/s10578-009-0153-x

Melo, A. I. M. T. (2005). Emoções no período escolar: Estratégias parentais face à expressão emocional e sintomas de internalização e externalização da criança (Dissertação de mestrado, Instituto de Educação e Psicologia da Universidade do Minho, Portugal). Recuperado em http://hdl.handle.net/1822/4926

Morgan, J. K., Izard, C. E., \& King, K. A. (2010). Construct validity of the emotion matching task: Preliminary evidence for convergent and criterion validity of a new emotion knowledge measure for young children. Social Development, 19(1), 52-70. doi:10.1111/j.1467-9507.2008.00529.x

Oliveira, S. E. S., \& Bandeira, D. R. (2011). Linguistic and cultural adaptation of the Inventory of Personality Organization (IPO) for the Brazilian culture. Journal of Depression and Anxiety, 1(1). doi:10.4172/2167-1044.1000105

Oliveira, S. S. G., Dias, M. G. B. B., \& Roazzi, A. (2003). O lúdico e suas implicações nas estratégias de regulação das emoções em crianças hospitalizadas. Psicologia: Reflexão e Crítica, 16(1), 1-13. doi:10.1590/S0102-79722003000100003

Pears, K. C., Fisher, P. A., \& Bronz, K. D. (2007). An intervention to promote social emotional school readiness in foster children: Preliminary outcomes from a pilot study. School Psychology Review, 36(4), 665-673.

Ramsden, S., \& Hubbard, J. (2002). Family Expressiveness and parental emotion coaching: Their role in children's emotion regulation and aggression. Journal of Abnormal Child Psychology, 30(6), 657-667. doi:10.1023/a: 1020819915881

Rocha, M. M., Ferrari, R. A., \& Silvares, E. F. M. (2011). Padrões de concordância entre múltiplos 
informantes na avaliação dos problemas comportamentais de adolescentes: Implicações clínicas. Estudos e Pesquisas em Psicologia, 11(3), 948-964. Recuperado em http://pepsic.bvsalud. org/scielo.php?script=sci_arttext\&pid=S1808$-42812011000300013 \& \operatorname{lng}=p t \& \operatorname{tng}=p t$

Rubin, K. H., Coplan, R. J., Fox, N. A., \& Calkins, S. D. (1995). Emotionality, emotion regulation, and preschoolers' social adaptation. Development and Psychopathology, 7(1), 49-62. doi:10.1017/S0954579400006337

Sandoval, J., \& Durán, R. P. (1998). Language. In J. Sandoval, C. L. Frisby, K. F. Geisinger, J. D. Sheuneman, \& J. R. Grenier (Eds.), Test interpretation and diversity: Achieving equity in assessment wording (pp. 181-211). Washington, DC: American Psychological Association.

Shapiro, A., \& Berge, J. F. (2002). Statistical inference of minimum rank factor analysis. Psychometrika, 67(1), 79-94. doi:10.1007/BF02294710

Shields, A. M., \& Cicchetti, D. (1995). The development of an emotion regulation assessment battery: Reliability and validity among at-risk grade-school children. Paper presented at the biennial meeting of the Society for Research on Child Development, Indianapolis, IN, USA.

Shields, A., \& Cicchetti, D. (1997). Emotion regulation among school age children: The development and validation of a new criterion q-sort scale. Developmental Psychology, 33(6), 906916. doi:10.1037/0012-1649.33.6.906

Shields, A., \& Cicchetti, D. (1998). Reactive aggression among maltreated children: The contributions of attention and emotion dysregulation. Journal of Clinical Child Psychology, 27(4), 381-395. doi:10.1207/s15374424jccp2704_2

Shields, A., \& Cicchetti, D. (2001). Parental maltreatment and emotion dysregulation as risk factors for bullying and victimization in middle childhood. Journal of Clinical Child \& Adolescent Psychology, 30(3), 349-363. doi:10.1207/ S15374424JCCP3003_7

Shields, A., Dickstein, S., Seifer, R., Giusti, L., Magee, K. D., \& Spritz, B. (2001). Emotional competence and early school adjustment: A study of preschoolers at risk. Early Education and Development, 12(1), 73-96. doi:10.1207/ s15566935eed1201_5
Shields, A., Ryan, R. M., \& Cicchetti, D. (2001). Narrative representations of caregivers and emotion dysregulation as predictors of maltreated children's rejection by peers. Developmental Psychology, 37(3), 321-337. doi:10.1037/00121649.37.3.321

Shipman, K., Edwards, A., Brown, A., Swisher, L., \& Jennings, E. (2005). Managing emotion in a maltreating context: A pilot study examining child neglect. Child Abuse and Neglect, 29(9), 1015-1029. doi:10.1016/j.chiabu.2005.01.006

Supplee, L. H., Skuban, E. M., Shaw, D. S., \& Prout, J. (2009). Emotion regulation strategies and later externalizing behavior among European American and African American children. Development and Psychopathology, 21(2), 393-415. doi:10.1017/S0954579409000224

Suveg, C., Kendall, P., Comer, J., \& Robin, J. (2006). Emotion-focused cognitive-behavioral therapy for anxious youth: A multiple-baseline evaluation. Journal of Contemporary Psychotherapy, 36(2), 77-85. doi:10.1007/s10879-006-9010-4

Toblin, R. L., Schwartz, D., Hopmeyer Gorman, A., \& Abou-ezzeddine, T. (2005). Social-cognitive and behavioral attributes of aggressive victims of bullying. Journal of Applied Developmental Psychology, 26(3), 329-346. doi:10.1016/j.appdev.2005.02.004

Trentacosta, C. J., \& Shaw, D. S. (2009). Emotional self-regulation, peer rejection, and antisocial behavior: Developmental associations from early childhood to early adolescence. Journal of Applied Developmental Psychology, 30(3), 356365. doi:10.1016/j.appdev.2008.12.016

Zeman, J., Shipman, K., \& Penza-Clyve, S. (2001). Development and initial validation of the Children's Sadness Management Scale. Journal of Nonverbal Behavior, 25(3), 187-205. doi:10.1023/a:1010623226626

Zeman, J. L., Cassano, M., Suveg, C., \& Shipman, K. (2010). Initial validation of the Children's Worry Management Scale. Journal of Child and Family Studies, 19(4), 381-392. doi:10.1007/ s10826-009-9308-4 
Apêndice

\section{Emotional Regulation Checklist (ERC) - Versão Brasileira}

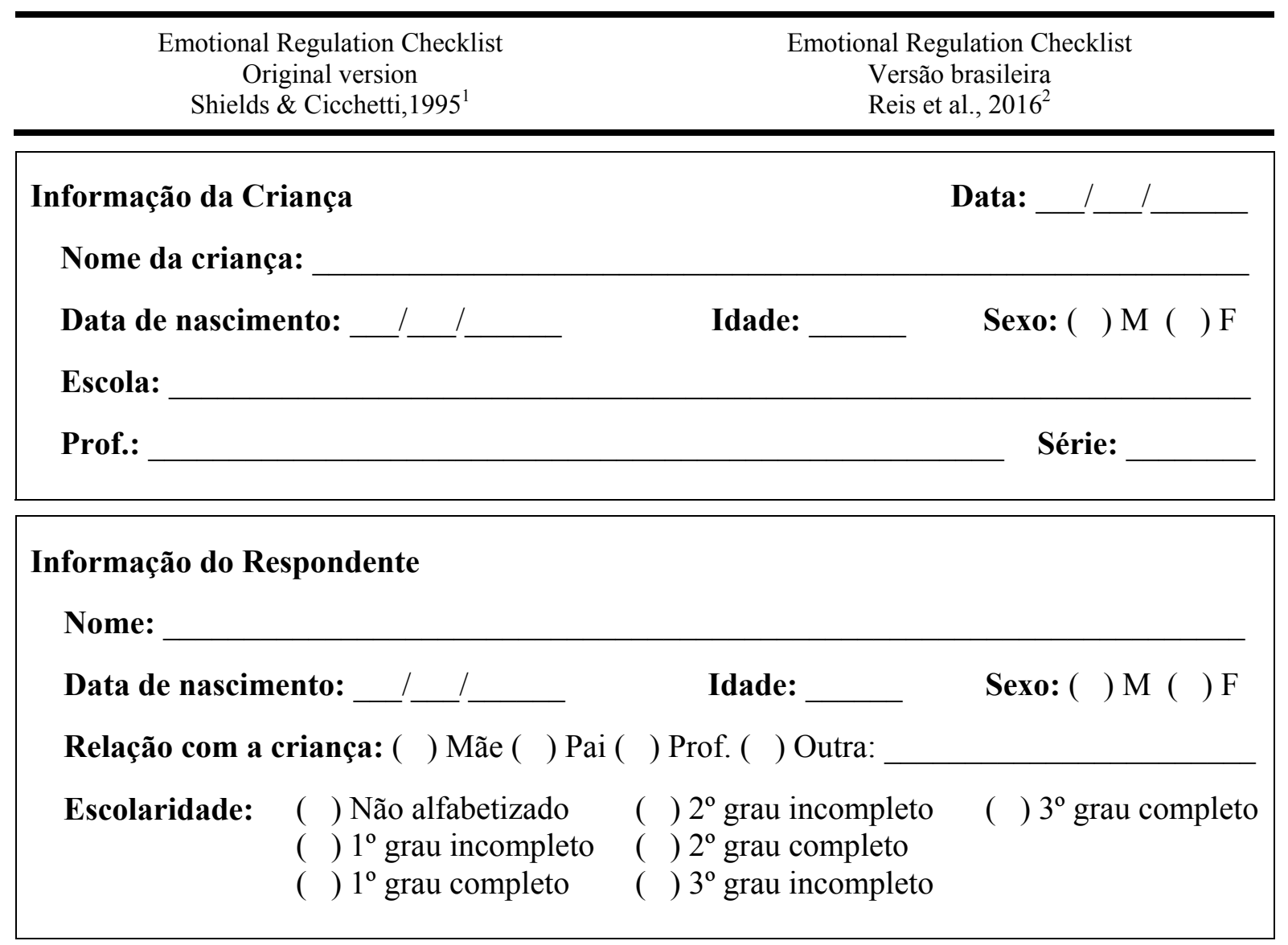

\section{Instrução:}

Por favor, leia as afirmações e coloque uma cruz $(X)$, ou um círculo $(\mathrm{O})$ na opção que melhor identifica aquilo que acontece com a criança que você está avaliando. Se você acha que a criança apresenta bastante do comportamento descrito na sentença marque "3" (muitas vezes) ou “4” (quase sempre), caso contrário, marque "1" (nunca) ou "2" (algumas vezes). Utilize a legenda para marcar as sentenças conforme você acha que os comportamentos descritos acontecem com a criança.

Legenda: 1 . Nunca $\mid$ 2. Algumas vezes $\mid$ 3. Muitas vezes $\mid$ 4. Quase sempre

\footnotetext{
${ }^{1}$ Shields, A. M., \& Cicchetti, D. (1995). The development of an emotion regulation assessment battery: Reliability and validity among at-risk grade-school children. Paper presented at the biennial meeting of the Society for Research on Child Development, Indianapolis.

${ }^{2}$ Reis, A. H., Oliveira, S. E. S., Bandeira, D. R., Andrade, N. C., Abreu, N., \& Sperb, T. M. (2016). Emotion Regulation Checklist (ERC): Preliminary studies of cross-cultural adaptation and validation for use in Brazil. Temas em Psicologia, 24(1), 63-82.
} 


\section{Emotional Regulation Checklist (ERC) - Versão Brasileira}

\begin{tabular}{|c|c|c|c|c|c|c|c|}
\hline 1. Nunca & 2. Algumas vezes & 3. Muitas vezes & \multicolumn{5}{|c|}{ 4. Quase sempre } \\
\hline \multicolumn{4}{|c|}{ 1. É uma criança alegre. } & 1 & 2 & 3 & 4 \\
\hline \multicolumn{4}{|c|}{$\begin{array}{l}\text { 2. Apresenta grande variação de humor (o estado emocional da criança é difícil de ser } \\
\text { previsto, pois ela muda rapidamente de bem humorada para mal humorada). }\end{array}$} & 1 & 2 & 3 & 4 \\
\hline \multicolumn{4}{|c|}{$\begin{array}{l}\text { 3. Responde de forma positiva a iniciativas de adultos de se aproximar de forma neutra ou } \\
\text { amigável. }\end{array}$} & 1 & 2 & 3 & 4 \\
\hline \multicolumn{4}{|c|}{$\begin{array}{l}\text { Troca bem de uma atividade para outra (não fica ansiosa, irritada, angustiada ou } \\
\text { excessivamente empolgada quando passa de uma atividade para outra). }\end{array}$} & 1 & 2 & 3 & 4 \\
\hline \multicolumn{4}{|c|}{$\begin{array}{l}\text { 5. Recupera-se rapidamente de episódios de aborrecimento ou angústia (por exemplo, não } \\
\text { permanece quieta ou mal humorada, ansiosa ou triste após eventos emocionalmente } \\
\text { estressantes). }\end{array}$} & 1 & 2 & 3 & 4 \\
\hline \multicolumn{4}{|c|}{ 6. Frustra-se facilmente. } & 1 & 2 & 3 & 4 \\
\hline \multicolumn{4}{|c|}{$\begin{array}{l}\text { 7. Responde de forma positiva a iniciativas de seus pares de se aproximar de forma neutra ou } \\
\text { amigável (pares são crianças da mesma idade ou colegas). }\end{array}$} & 1 & 2 & 3 & 4 \\
\hline \multicolumn{4}{|c|}{ 8. É propensa a explosões de raiva / birra. } & 1 & 2 & 3 & 4 \\
\hline & 1 & 2 & 3 & 4 \\
\hline \multicolumn{4}{|c|}{$\begin{array}{l}\text { 10. Sente prazer com o sofrimento dos outros (por exemplo, ri quando outra pessoa se machuca } \\
\text { ou é punida; gosta de provocar os outros). }\end{array}$} & 1 & 2 & 3 & 4 \\
\hline \multicolumn{4}{|c|}{$\begin{array}{l}\text { 11. Consegue controlar a empolgação em situações emocionalmente estimulantes (por } \\
\text { exemplo, não se empolga excessivamente em situações de brincadeiras de alta energia, ou não } \\
\text { fica muito agitada em contextos inapropriados). }\end{array}$} & 1 & 2 & 3 & 4 \\
\hline \multicolumn{4}{|c|}{ 12. É chorona ou gosta de ficar "agarrada" com adultos. } & 1 & 2 & 3 & 4 \\
\hline \multicolumn{4}{|c|}{ 13. É propensa a explosões inadequadas de animação e entusiasmo. } & 1 & 2 & 3 & 4 \\
\hline \multicolumn{4}{|c|}{ 14. Responde com raiva ou de forma zangada quando os adultos lhe impõem limites. } & 1 & 2 & 3 & 4 \\
\hline \multicolumn{4}{|c|}{$\begin{array}{l}\text { 15. A criança consegue dizer quando está se sentindo triste, com raiva ou zangada, com medo } \\
\text { ou assustada. }\end{array}$} & 1 & 2 & 3 & 4 \\
\hline \multicolumn{4}{|c|}{ 16. Parece triste ou apática. } & 1 & 2 & 3 & 4 \\
\hline \multicolumn{4}{|c|}{ 17. É excessivamente empolgada ao tentar envolver outros na brincadeira. } & 1 & 2 & 3 & 4 \\
\hline \multicolumn{4}{|c|}{$\begin{array}{l}\text { 18. Mostra humor apático (fisionomia é vaga e inexpressiva; a criança parece ausente } \\
\text { emocionalmente). }\end{array}$} & 1 & 2 & 3 & 4 \\
\hline \multicolumn{4}{|c|}{$\begin{array}{l}\text { 19. Responde de forma negativa a aproximações neutras ou amigáveis feitas por pares } \\
\text { (crianças ou colegas da mesma idade) (por exemplo, pode falar com um tom de voz com raiva } \\
\text { ou responder com medo). }\end{array}$} & 1 & 2 & 3 & 4 \\
\hline \multicolumn{4}{|l|}{ 20. É impulsiva. } & 1 & 2 & 3 & 4 \\
\hline \multicolumn{4}{|c|}{$\begin{array}{l}\text { 21. É empática com os outros. Mostra preocupação quando os outros estão chateados ou } \\
\text { angustiados. }\end{array}$} & 1 & 2 & 3 & 4 \\
\hline \multicolumn{4}{|c|}{ 22. Demonstra entusiasmo que os outros consideram inapropriado, intrusivo ou intrometido. } & 1 & 2 & 3 & 4 \\
\hline \multicolumn{4}{|c|}{$\begin{array}{l}\text { 23. Mostra emoções negativas apropriadas (raiva, medo, frustração, angústia) em resposta a } \\
\text { atos hostis, agressivos ou intrusivos dos pares (crianças ou colegas da mesma idade). }\end{array}$} & 1 & 2 & 3 & 4 \\
\hline \multicolumn{4}{|l|}{24.} & & & & \\
\hline
\end{tabular}




\section{Emotional Regulation Checklist (ERC) - Versão Brasileira}

\section{Apuração:}

1. Registre o escore dado na coluna da esquerda para cada item.

2. Registre os escores para cada subescala. Para a apuração das subescalas alguns itens precisam ser invertidos. Eles estão sinalizados com a abreviação (Inv). Considere a inversão dos itens da seguinte forma $(1=4,2=3,3=2$ e $4=1)$, sempre tendo como referência o escore registrado na primeira coluna da esquerda. Preencha somente os espaços em branco.

3. Some o total para cada subescala.

\begin{tabular}{|c|l|}
\hline Item & Escore \\
\hline 1 & \\
\hline 2 & \\
\hline 3 & \\
\hline 4 & \\
\hline 5 & \\
\hline 6 & \\
\hline 7 & \\
\hline 8 & \\
\hline 9 & \\
\hline 10 & \\
\hline 11 & \\
\hline 12 & \\
\hline 13 & \\
\hline 14 & \\
\hline 15 & \\
\hline 16 & \\
\hline 17 & \\
\hline 18 & \\
\hline 19 & \\
\hline 20 & \\
\hline 21 & \\
\hline 22 & \\
\hline 23 & \\
\hline 24 & \\
\hline & \\
\hline & \\
\hline
\end{tabular}
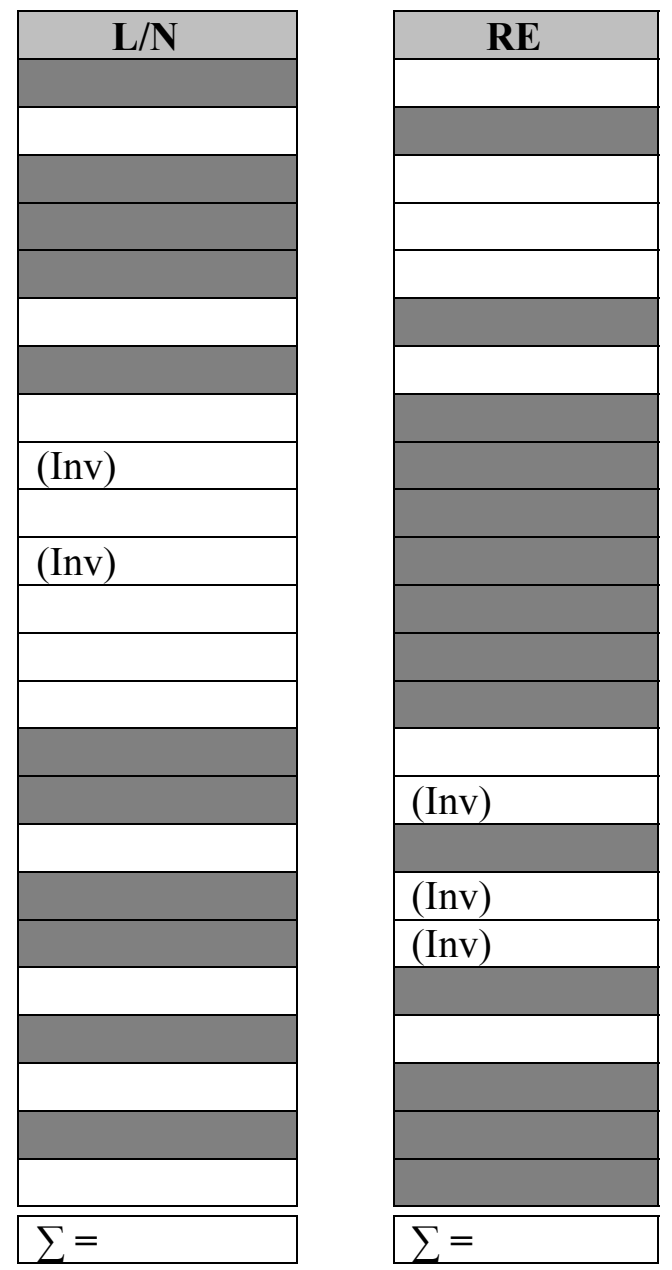

\begin{tabular}{|l|}
\hline \multicolumn{1}{|c|}{ RE Total } \\
\hline (Inv) \\
\hline \\
\hline (Inv) \\
\hline (Inv) \\
\hline (Inv) \\
\hline (Inv) \\
\hline (Inv) \\
\hline (Inv) \\
\hline (Inv) \\
\hline (Inv) \\
\hline (Inv) \\
\hline (Inv) \\
\hline (Inv) \\
\hline (Inv) \\
\hline (Inv) \\
\hline$\sum=$ \\
\hline
\end{tabular}

\section{Interpretação:}

Ainda não foram conduzidos estudos normativos.

\section{Permissão para uso do instrumento:}

Não há necessidade de solicitar permissão para o uso do presente instrumento. É exigido, contudo, que o devido crédito seja concedido aos seus autores. O presente artigo deve ser utilizado para a citação do instrumento, e esta nota assegura a permissão para sua utilização. 